\section{Why Michael Couldn't Hit:}

\section{AND OTHER TALES OF THE NEUROLOGY OF SPORTS}

\author{
by Harold L. Klawans \\ W.H. Freeman and Company \\ 1996 ISBN: 0-7167-3001-4 \$22.95
}

REVIEWED BY SIR ROGER BANNISTER

Hon. Consultant Neurologist to Queen's Square

and St. Mary's Hospital, London, UK

former Master of Pembroke College Oxford, UK

Sport can cause great exhilaration and good health but, sadly, is also a cause of mental and physical affliction. The stress sport engenders can be subject to more precise medical observation than the famous remark by the great American coach Yogi Berra, "Baseball is $90 \%$ mental and the other half is physical." Harold Klawans' book is an eclectic mixture of anecdote, history and neurology - his own profession.

Some sporting problems are simply mechanical. Man, we are told, evolved from a

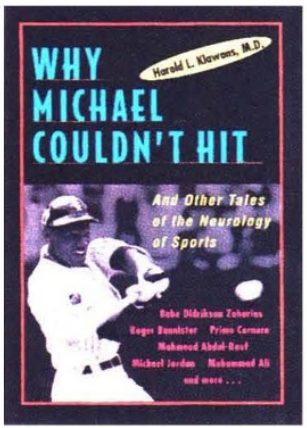

might have caused a bitemporal field defect that prevented him from seeing clearly the origin of blows aimed at him. My own view of boxing is that no civilized country should tolerate this "sport," in which the deliberate intent is to inflict damage on the body's most delicate organ - the brain. Muhammad Ali developed Parkinson's disease, probably because of repeated trauma, and survived. Many boxers have developed progressive dementia or strokes due to cerebral hemorrhage or have even been killed.

Of current interest is the basketball player Mahmoud Abdul-Rauf with the genetically determined Tourette syndrome, in which scatological utterances are linked with motor tics - but never when the victim is concentrating - and an obsessional personality disorder. The obsessional feature enabled Rauf to train endlessly, refusing to quit until he had sunk a straight set of 10 "nothing but net" baskets from a free-throw position.

For a neurologist the greatest interest in this book lies in its discussion of the complex strain sport places on the brain's capacity to learn complex tasks, different but in some ways resembling language and music skills that are more easily learned when young. A fourminute piano performance of quadruped and the human shoulder is ill-adapted to throwing a baseball at a hundred miles per hour. The thrower may trap his supraspinatus nerve, as the pitcher Bruce Sutter did, or block his brachial artery, as the pitcher J. R. Richard did. Sometimes, of course, athletes will have incidental neurological diseases, and in these cases, Klawans gives us useful tutorials. Amyotrophic lateral sclerosis (ALS) is sometimes called Lou Gehrig's disease in America. Myasthenia causes fatigue on hard exercise, which is why Dwight Jameson was nicknamed "First-Half Jameson." The sprinter Wilma Rudolph, described as "flying like a butterfly," overcame poliomyelitis and a leg brace in childhood.

Of more interest are the diseases that seem actually to have contributed to sporting success. The Italian boxer Primo Carnera, who was discovered in a French boxing booth, was an acromegalic with a pituitary tumor causing gigantism. At 6 feet 7 inches with an arm span of 7 feet, this disease gave him weight and reach, with enormous hands and feet, but without great muscle strength or coordination. As a neurologist, I wonder whether, in addition, his pituitary tumor over five thousand notes requires some 70 manual finger movements a second. A pole vaulter does a reverse twist handstand on a moving 15 -foot pole while running at 20 miles per hour. The title of the book comes from a description of how the world's greatest basketball player, Michael Jordan, tried at the age of 31 to switch to baseball and failed. His skills were just not transferable. However, some athletes are versatile from the start. Babe Didrikson won five US national championship titles and three Olympic medals in track and field before becoming the world's greatest woman golfer. Are such athletes genetically programmed to have exceptional adaptable skills, in particular in perceiving the speed and direction of moving balls they catch or hit?

When complex neurological coordination goes wrong it is difficult to control. The golfer Ben Hogan very publicly lost the ability to putt because of an involuntary jerk popularly known as the "yips." His case is more complicated in that as a left-handed boy he was too poor to buy left-handed clubs so he had to play righthanded. As the game is said to require subordination of the normally dominant hand, this might have helped his swing

up to a point, but did the mental strain of the transfer make him more vulnerable to later breakdown?

I am puzzled and indeed slightly embarrassed to find myself included in this book rather as a grace note because I happen to be a neurologist with a past in sport. In public I will admit to no neurological oddities beyond that degree of obsessiveness desirable in sport and also, I may add as Klawans well knows, a prerequisite for a successful neurological career!

The serious message of the book is that all learning is complex and difficult, whether academic or sporting, and the moment to learn differs for each activity and must be exploited early for success. Klawans is to be congratulated. I hope his love of neurology does not dampen his ardor for writing.

\title{
Molecular Mechanisms of Dementia
}

\author{
edited by Wilma Wasco \\ and Rudolph E. Tanzi \\ The Humana Press, 1996 \\ ISBN: 0-896-03371-6\$99.50
}

Reviewed by Allen D. Roses

Jefferson Pilot Corporation Professor of Neurobiology and Neurology Chief, Division of Neurology

227 Bryan Research Building, Research Drive Duke University Medical Center

Durham, North Carolina 27710, USA

Molecular Mechanisms of Dementia is directed toward scientists with a background in neurobiology and an interest in

Molecular

Mechanisms

of Dementia

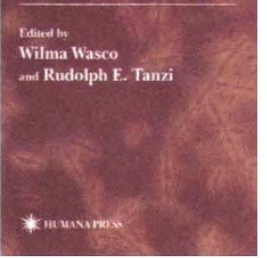

dementia. Its eighteen chapters are meant to span the field of Alzheimer's disease $(\mathrm{AD})$ as well as more general areas thought to be related to dementia. If the coverage of dementia followed the chapter titles and each provided an introduction to the area and its relevance to the field, most neurobiologists would find this book very useful and interesting. Unfortunately, the interrelationships among the chapters are not clear, so that the book reads as a compilation of approaches. The context and status of each approach within the field of 
dementia is poorly defined for the general reader. If the number of pages per field of emphasis reflects relative importance, then the book stresses certain areas very heavily and ignores other major themes of neurobiology related to the dementias.

Any multi-authored compilation runs the risk of being unbalanced. The title of this book implies a cohesive and balanced overview of the neurobiology of dementia. Indeed, several of the chapters are excellent and provide relative placement within the field and an interesting and thoughtful perspective on dementia. The chapter "Calcium Homeostasis and Free Radical Metabolism as Convergence Points in the Pathophysiology of Dementia," is one of the longer contributions and should be read by anyone interested in this field. "Prion Diseases and Dementia" provides an excellent clinical, pathological and genetic overview of these diseases. However, several of the chapters concentrate on authors' pet theories, giving very short shrift to other considerations.

The read is easier if one is already familiar with the field of dementia. A fivepage, well-written summary of the anatomy of $\mathrm{AD}$ may be sufficient for a reader immersed in the field, but a more complete comparative anatomy of $\mathrm{AD}$ and other dementias may be more helpful to the general audience. There are no fewer than five chapters that deal with the role of the amyloid $\beta$-protein $(A \beta)$ precursor (APP) or the putative effects of $A \beta$. Each of these chapters is based on the fundamental hypothesis that the deposition of the A $\beta$ fragment of APP causes AD. Although this reflects the interests of the editors, it is not balanced by inclusion of proponents of several alternative viewpoints on pathogenesis. The editors state that "the contribution of genetic strategies has been fundamental to discovery," and they are correct. However, most of AD is not due to autosomal dominant mutations (defined or remaining unknown) and, for example, a growing field of neurobiology related directly to the brain metabolism of apolipoprotein $\mathrm{E}$ is summarized in a single chapter with one point of view.

Gene discovery in the defined forms of $\mathrm{AD}$ also provides a means of predicting which groups of patients are most likely to become afflicted with AD. A growing and important literature germane to discussions of molecular mechanisms is emerging from the combined application of genetics and epidemiology to temporal questions of disease onset and pathogenesis. It is certainly difficult to tell what occurs first when only post-mortem material is considered. However, it is now possible to predict which groups of individuals are at risk and to apply a range of metabolic and anatomical analyses to study their temporal relationship with onset of dementia. For example, positron emission tomography studies of groups of unaffected individuals who are at an increased genetic risk for $\mathrm{AD}$ have documented decreased oxygen metabolism in areas of the brain characteristic of $\mathrm{AD}$. These metabolic changes occur more than two decades before the median age of onset of disease, well before significant $A \beta$ deposition has occurred. Unfortunately, the role of genetics combined with functional imaging, earliest neuropathology, epidemiology and other exciting emerging fields is barely mentioned in this text.

The book contains several superb chapters by authors with defined viewpoints and these are interesting and provocative reading for scientists in the field of dementia. However, as a general reference to be read cover to cover by interested neuroscientists, the volume is unbalanced. No book of this length could hope to be complete, but a balanced distribution of content within context would make the book more accessible to the general neurobiologist.

\section{Concepts in Vaccine Development}

\section{edited by Stefan H.E. Kaufmann Walter de Gruyter \& Co. ISBN: 3-11-014815-3 \$119.00}

REVIEWEd BY LOUIS D. FALO, JR. Assistant Professor and Vice Chairman Department of Dermatology

University of Pittsburgh School of Medicine

University of Pittsburgh Cancer Institute

Pittsburgh, Pennsylvania 15213, USA

Recent advances in several disciplines have resulted in significant progress in vaccine design. "Vaccinology" has evolved from a primarily empirical art to a hypothesis-driven science drawing on advances in molecular genetics, cell biology, protein chemistry and immunology. Vaccine design is now the focus of multidisciplinary teams of investigators in academia and of "core technology platforms" in the private sector. It is perhaps this influx of new investigators and the convergence of established investigators with diverse scientific backgrounds that has created a need for a book that can serve as a single-source, comprehensive overview of vaccinology. Concepts in Vaccine Development, successfully addresses this need.

This book aims to provide the reader with a state-of-the-art overview of "all aspects of vaccinology from the bench to the field." As a collection of reviews, its breadth is certainly impressive, beginning with a bicentennial tribute to the first vaccination by Edward Jenner and ending with a lucid review of the application of vaccination therapy to autoimmune disease. The 53 contributors (to 22 chapters) provide a systematic review of critical issues in a nearly manageable 570 pages. As is the case with most books of this type, individual chapters vary in quality. The inclusion of so many topics means that several chapters are somewhat limited in scope. Similarly, it is rare for such books to offer the most up-to-date information, particularly in areas where progress has been so rapid. However, the real strength of this text is not in the chapters dealing with subjects with which the reader may be familiar, but in the chapters on less familiar topics. Most would serve as excellent starting points for ventures into new areas of investigation. Most important, by presenting quality reviews on diverse concepts, this book provides the reader with a genuine sense of perspective.

The book is divided into four sections. The first appropriately establishes the need for new vaccines, addressing the issue from evolutionary, economic and public health perspectives. A review addressing the economics of vaccination presents economic concepts and analysis in a manner amenable to non-economists and includes a particularly compelling discussion of the use of "cost-effectiveness" analysis to identify priorities in vaccine research.

The other three sections are oriented more toward basic research, and the reader can readily sense the renewed optimism that has permeated this field. The section "General Principles of Immunology" introduces concepts in the context of strategies for vaccine design against intracellular bacteria, viruses and Leishmania parasites. The chapter on viral vaccine strategies ends with a general consideration of cytotoxic T-lymphocyte priming and immunodominance and nicely complements the fol- 